\title{
Oxides and nitrides as alternative plasmonic materials in the optical range [Invited]
}

Naik, Gururaj V.; Kim, Jongbum; Boltasseva, Alexandra

Published in:

Optical Materials Express

Link to article, DOI:

10.1364/OME.1.001090

Publication date:

2011

Document Version

Publisher's PDF, also known as Version of record

Link back to DTU Orbit

Citation (APA):

Naik, G. V., Kim, J., \& Boltasseva, A. (2011). Oxides and nitrides as alternative plasmonic materials in the optical range: [Invited]. Optical Materials Express, 1(6), 1090-1099. https://doi.org/10.1364/OME.1.001090

\section{General rights}

Copyright and moral rights for the publications made accessible in the public portal are retained by the authors and/or other copyright owners and it is a condition of accessing publications that users recognise and abide by the legal requirements associated with these rights.

- Users may download and print one copy of any publication from the public portal for the purpose of private study or research.

- You may not further distribute the material or use it for any profit-making activity or commercial gain

- You may freely distribute the URL identifying the publication in the public portal 


\title{
Oxides and nitrides as alternative plasmonic materials in the optical range [Invited]
}

\author{
Gururaj V. Naik, ${ }^{1}$ Jongbum Kim, ${ }^{1}$ and Alexandra Boltasseva ${ }^{1,2,3, *}$ \\ ${ }^{1}$ Birck Nanotechnology Center and School of Electrical \& Computer Engineering, \\ Purdue University, West Lafayette, Indiana 47906, USA \\ ${ }^{2}$ DTU Fotonik, Technical University of Denmark, Lyngby 2800, Denmark \\ ${ }^{3}$ Erlangen Graduate School in Advanced Optical Technologies (SAOT), \\ Friedrich-Alexander-Universität Erlangen-Nürnberg, 91052 Erlangen, Germany \\ *aeb@purdue.edu
}

\begin{abstract}
As alternatives to conventional metals, new plasmonic materials offer many advantages in the rapidly growing fields of plasmonics and metamaterials. These advantages include low intrinsic loss, semiconductor-based design, compatibility with standard nanofabrication processes, tunability, and others. Transparent conducting oxides such as $\mathrm{Al}: \mathrm{ZnO}, \mathrm{Ga}: \mathrm{ZnO}$ and indium-tin-oxide (ITO) enable many high-performance metamaterial devices operating in the near-IR. Transition-metal nitrides such as TiN or $\mathrm{ZrN}$ can be substitutes for conventional metals in the visible frequencies. In this paper we provide the details of fabrication and characterization of these new materials and discuss their suitability for a number of metamaterial and plasmonic applications.
\end{abstract}

(C) 2011 Optical Society of America

OCIS codes: (160.3918) Metamaterials; (160.4236) Nanomaterials; (250.5403) Plasmonics; (310.6860) Thin films, optical properties.

\section{References and links}

1. A. Boltasseva and H. Atwater, "Low-loss plasmonic metamaterials," Science 331, 290-291 (2011).

2. S. Kehr, Y. Liu, L. Martin, P. Yu, M. Gajek, S. Yang, C. Yang, M. Wenzel, R. Jacob, H. von Ribbeck, M. Helm, X. Zhang, L. Eng, and R. Ramesh, "Near-field examination of perovskite-based superlenses and superlensenhanced probe-object coupling," Nat. Commun. 2, 249-249 (2011).

3. J. Luther, P. Jain, T. Ewers, and A. Alivisatos, "Localized surface plasmon resonances arising from free carriers in doped quantum dots," Nat. Mater. 10, 361-366 (2011).

4. D. Slocum, S. Inampudi, D. Adams, S. Vangala, N. Kuhta, W. Goodhue, V. Podolskiy, and D. Wasserman, "Funneling light through a subwavelength aperture with epsilon-near-zero materials," Arxiv preprint arXiv:1103.6013 (2011).

5. E. Feigenbaum, K. Diest, and H. Atwater, "Unity-order index change in transparent conducting oxides at visible frequencies," Nano Lett. 10, 2111-2116 (2010).

6. D.-G. Park, T.-H. Cha, K.-Y. Lim, H.-J. Cho, T.-K. Kim, S.-A. Jang, Y.-S. Suh, V. Misra, I.-S. Yeo, J.-S. Roh, J. W. Park, and H.-K. Yoon, "Robust ternary metal gate electrodes for dual gate CMOS devices," in "Electron Devices Meeting, 2001. IEDM Technical Digest. International,” (IEEE, 2001), pp. 30.6.1-30.6.4.

7. P. West, S. Ishii, G. Naik, N. Emani, V. Shalaev, and A. Boltasseva, "Searching for better plasmonic materials," Laser Photon. Rev. 4, 795-808 (2010).

8. D. Bobb, G. Zhu, M. Mayy, A. Gavrilenko, P. Mead, V. Gavrilenko, and M. Noginov, "Engineering of low-loss metal for nanoplasmonic and metamaterials applications," Appl. Phys. Lett. 95, 151102 (2009).

9. G. Naik and A. Boltasseva, "Ceramic plasmonic components for optical metamaterials," in "Quantum Electronics and Laser Science Conference" (Optical Society of America, 2011).

\#151151 - \$15.00 USD Received 15 Jul 2011; revised 29 Aug 2011; accepted 30 Aug 2011; published 6 Sep 2011

(C) 2011 OSA

1 October 2011 / Vol. 1, No. 6 / OPTICAL MATERIALS EXPRESS 1090 
10. G. Zhu, L. Gu, J. Kitur, A. Urbas, J. Vella, and M. Noginov, "Organic materials with negative and controllable electric permittivity," in "Quantum Electronics and Laser Science Conference" (Optical Society of America, 2011).

11. G. V. Naik and A. Boltasseva, "Semiconductors for plasmonics and metamaterials," Phys. Status Solidi (RRL) 4, 295-297 (2010).

12. N. Ashcroft and N. Mermin, Solid State Physics (Saunders College, 1976).

13. M. Yoon, S. Lee, H. Park, H. Kim, and M. Jang, "Solid solubility limits of Ga and Al in ZnO," J. Mater. Sci. Lett. 21, 1703-1704 (2002).

14. K. Tominaga, H. Manabe, N. Umezu, I. Mori, T. Ushiro, and I. Nakabayashi, "Film properties of ZnO: Al prepared by cosputtering of ZnO:Al and either Zn or Al targets," J. Vac. Sci. Technol. A 15, 1074-1079 (1997).

15. K. Ellmer and R. Mientus, "Carrier transport in polycrystalline ITO and ZnO:Al II: the influence of grain barriers and boundaries," Thin Solid Films 516, 5829-5835 (2008).

16. D. Horwat, M. Jullien, F. Capon, J. Pierson, J. Andersson, and J. Endrino, "On the deactivation of the dopant and electronic structure in reactively sputtered transparent Al-doped ZnO thin films," J. Phys. D: Appl. Phys. 43, 132003 (2010).

17. K. Kim, K. Park, and D. Ma, "Structural, electrical and optical properties of aluminum doped zinc oxide films prepared by radio frequency magnetron sputtering," J. Appl. Phys. 81, 7764-7772 (1997).

18. D. Kim, M. Park, H. Lee, and G. Lee, "Thickness dependence of electrical properties of ITO film deposited on a plastic substrate by RF magnetron sputtering," Appl. Surf. Sci. 253, 409-411 (2006).

19. A. Suzuki, M. Nakamura, R. Michihata, T. Aoki, T. Matsushita, and M. Okuda, "Ultrathin Al-doped transparent conducting zinc oxide films fabricated by pulsed laser deposition," Thin Solid Films 517, 1478-1481 (2008).

20. M. Lee, J. Lim, J. Bang, W. Lee, and J. Myoung, "Effect of the thickness and hydrogen treatment on the properties of Ga-doped ZnO transparent conductive films," Appl. Surf. Sci. 255, 3195-3200 (2008).

21. T. Minami, T. Miyata, Y. Ohtani, and T. Kuboi, "Effect of thickness on the stability of transparent conducting impurity-doped ZnO thin films in a high humidity environment," Phys. Status Solidi (RRL) 1, R31-R33 (2007).

22. B. Karlsson, R. Shimshock, B. Seraphin, and J. Haygarth, "Optical properties of CVD-coated TiN, ZrN and HfN," Phys. Scripta 25, 775-779 (1982).

23. W.-C. Chen, Y.-R. Lin, X.-J. Guo, and S.-T. Wu, "Heteroepitaxial TiN of very low mosaic spread on $\mathrm{Al}_{2} \mathrm{O}_{3}$," Jpn. J. Appl. Phys. 42, 208-212 (2003).

24. B. Johansson, J. Sundgren, J. Greene, A. Rockett, and S. Barnett, "Growth and properties of single crystal TiN films deposited by reactive magnetron sputtering,” J. Vac. Sci. Technol. A 3, 303-307 (1985).

25. P. Patsalas and S. Logothetidis, "Optical, electronic, and transport properties of nanocrystalline titanium nitride thin films," J. Appl. Phys. 90, 4725-4734 (2001).

26. N. Fang, H. Lee, C. Sun, and X. Zhang, "Sub-diffraction-limited optical imaging with a silver superlens," Science 308, 534-537 (2005).

27. Z. Liu, H. Lee, Y. Xiong, C. Sun, and X. Zhang, "Far-field optical hyperlens magnifying sub-diffraction-limited objects," Science 315, 1686-1686 (2007).

28. D. Schurig, J. Mock, B. Justice, S. Cummer, J. Pendry, A. Starr, and D. Smith, "Metamaterial electromagnetic cloak at microwave frequencies," Science 314, 977-980 (2006).

29. A. Kildishev and V. Shalaev, "Engineering space for light via transformation optics," Opt. Lett. 33, 43-45 (2008).

30. P. Johnson and R. Christy, "Optical constants of the noble metals," Phys. Rev. B 6, 4370-4379 (1972).

31. G. Naik and A. Boltasseva, "A comparative study of semiconductor-based plasmonic metamaterials," Metamaterials 5, 1-7 (2011).

32. M. Noginov, L. Gu, J. Livenere, G. Zhu, A. Pradhan, R. Mundle, M. Bahoura, Y. Barnakov, and V. Podolskiy, "Transparent conductive oxides: plasmonic materials for telecom wavelengths," Appl. Phys. Lett. 99, 021101 (2011).

33. V. Drachev, U. Chettiar, A. Kildishev, H. Yuan, W. Cai, and V. Shalaev, "The Ag dielectric function in plasmonic metamaterials," Opt. Express 16, 1186-1195 (2008).

34. G. Naik, J. Schroeder, T. Sands, and A. Boltasseva, "Titanium nitride as a plasmonic material for visible wavelengths," Arxiv preprint arXiv:1011.4896 (2010).

35. E. Narimanov and A. Kildishev, "Optical black hole: broadband omnidirectional light absorber," Appl. Phys. Lett. 95, 041106 (2009).

36. H. Kim, J. Horwitz, S. Qadri, and D. Chrisey, "Epitaxial growth of Al-doped ZnO thin films grown by pulsed laser deposition," Thin Solid Films 420, 107-111 (2002).

37. B. Lee, T. Kim, and S. Jeong, "Growth and characterization of single crystalline Ga-doped ZnO films using RF magnetron sputtering," J. Phys. D: Appl. Phys. 39, 957-961 (2006).

38. A. Gālca, M. Secu, A. Vlad, and J. Pedarnig, "Optical properties of zinc oxide thin films doped with aluminum and lithium," Thin Solid Films 518, 4603-4606 (2010).

\#151151 - \$15.00 USD Received 15 Jul 2011; revised 29 Aug 2011; accepted 30 Aug 2011; published 6 Sep 2011

(C) 2011 OSA

1 October 2011 / Vol. 1, No. 6 / OPTICAL MATERIALS EXPRESS 1091 


\section{Introduction}

The introduction of new materials into the realm of plasmonics and metamaterials (MMs) is expanding the application domain of feasible devices [1]. Recent demonstrations of superlensing in the mid-IR [2], semiconductor plasmonic quantum dots [3] and an epsilon-near-zero (ENZ) light funnel [4] are but a few examples. The integration of new materials not only opens up possibilities for new devices, but it also significantly improves the performance of many existing MM and plasmonic devices. One of the most important challenges in the fields of plasmonics and MMs is the high loss in the metallic components of a device. New plasmonic materials have the promise of overcoming this major bottle-neck and enabling high-performance devices. Also, new plasmonic materials allow greater flexibility in the design of a device owing to the moderate magnitude of the real part of permittivity in such materials. On the contrary, metals such as gold and silver have very large negative real permittivities in the near-IR and visible ranges, which is a major obstacle in the design and fabrication of efficient devices. Alternative plasmonic materials have two other major advantages: they can exhibit tunable optical properties [5], and they can be compatible with standard fabrication and integration procedures [6]. Clearly, alternative plasmonic materials have significant advantages over conventional metals for plasmonic and metamaterial designs.

Alternative plasmonic materials in the near-IR and visible ranges can be classified into categories such as semiconductor-based [7], intermetallics [8], ceramics [9] and organic materials [10]. In this article, we show that inorganic ceramic materials: semiconductor-based oxides and transition-metal nitrides can be alternative plasmonic materials in the near-IR and visible ranges respecively. These materials have advantages over the other types, since oxides enable low-loss all-semiconductor based plasmonic and MM devices in the near-IR, while metal-nitrides are CMOS compatible and provide alternatives to gold and silver in the visible frequencies. Here, we describe our fabrication methods for these materials and study their optical properties in the context of plasmonic and MM applications. A brief discussion on the suitability of these new materials for various plasmonic and MM devices is also presented.

\section{Processing and characterization}

Whether we realize it or not, oxide plasmonic materials are a familiar element in everyday life since transparent conducting oxides (TCOs) are regularly used in liquid-crystal displays. These materials can exhibit metallic properties in the near-IR when heavily doped [11]. Doping produces high carrier concentration $\left(N>10^{20} \mathrm{~cm}^{-3}\right)$ which results in large plasma frequency $\left(\omega_{p} \propto \sqrt{N}\right)$. A large plasma frequency results in Drude-metal-like optical properties [12]. The following equation describes the Drude response of such degenerately doped semiconductors:

$$
\varepsilon=\varepsilon^{\prime}+i \varepsilon^{\prime \prime}=\varepsilon_{\infty}-\frac{\omega_{p}^{2}}{\omega(\omega+i \gamma)} .
$$

$\varepsilon_{\infty}$ is due to the screening effect of bound electrons in the material and can be considered as a constant in the frequency range of interest. $\gamma$ is the Drude-relaxation rate or damping coefficient of free carriers. This term signifies the optical losses incurred in the material. In order to have negative $\varepsilon^{\prime}$ in the optical range, $\omega_{p}$ must be large and $\varepsilon_{\infty}$ must be small. Lower losses require smaller $\gamma$. Heavy doping of $10^{21} \mathrm{~cm}^{-3}$ poses problems due to solid-solubility limits in many semiconductors. However, oxide semiconductors such as zinc oxide and indium oxide overcome this problem [13] and can be heavily doped to be metallic substitutes in the near-IR. Further increases of the carrier concentration to about $10^{22} \mathrm{~cm}^{-3}$ are required to make metal substitutes in the visible range; at those concentrations, oxides also suffer from solid-solubility limits. However, transition-metal nitrides can posses such high carrier concentrations and there-

\#151151 - \$15.00 USD Received 15 Jul 2011; revised 29 Aug 2011; accepted 30 Aug 2011; published 6 Sep 2011

(C) 2011 OSA

1 October 2011 / Vol. 1, No. 6 / OPTICAL MATERIALS EXPRESS 1092 

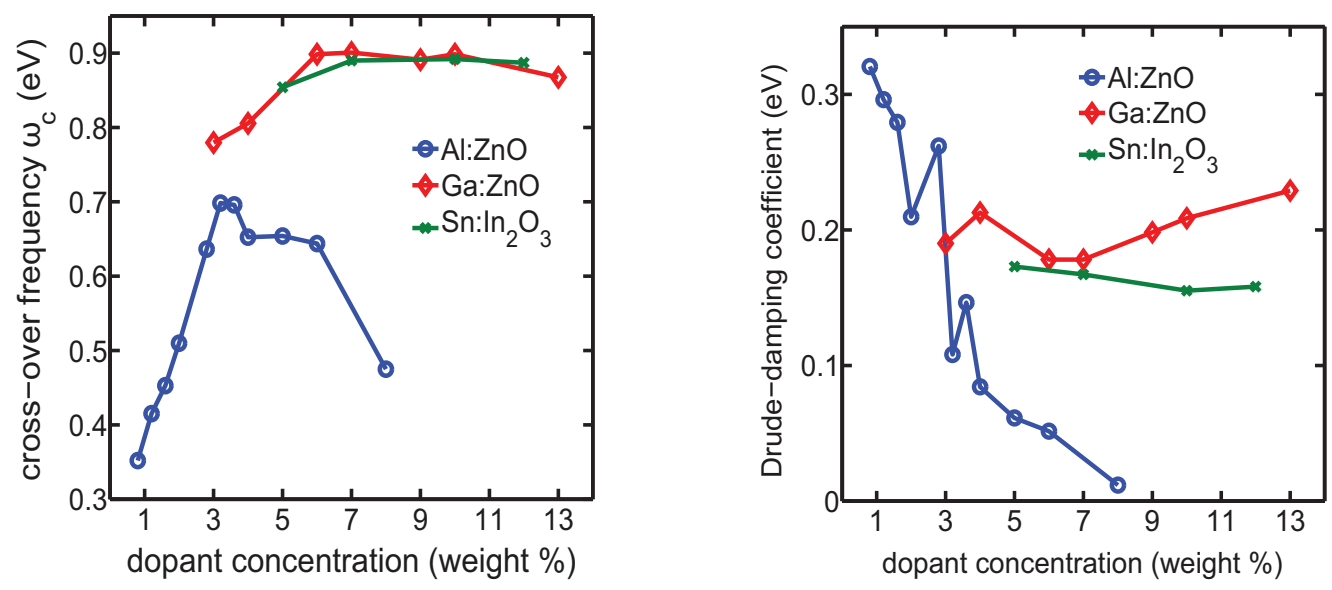

Fig. 1. Left panel: Cross-over frequency (frequency at which real permittivity crosses zero) of $\mathrm{Al}: \mathrm{ZnO}$, ITO and $\mathrm{Ga}: \mathrm{ZnO}$ films as a function of dopant concentration. Right panel: Drude-damping coefficient $(\gamma)$ vs. dopant concentration. The films were deposited at 100 ${ }^{\circ} \mathrm{C}$ (AZO and ITO) and $50{ }^{\circ} \mathrm{C}$ (GZO) with oxygen partial pressures of 0.4 mTorr. The ablation energy was about $2 \mathrm{~J} / \mathrm{cm}^{2}$.

fore can exhibit metallic properties at visible frequencies. In this work, we have studied the optical properties of aluminum-doped zinc oxide (AZO), gallium-doped zinc oxide (GZO), indium-tin-oxide (ITO) and nitrides of titanium, tantalum, hafnium and zirconium.

\subsection{Transparent conducting oxides}

Thin films of TCOs can be deposited by many physical-vapor and chemical-vapor deposition techniques. Highly conductive TCO films can be produced by techniques such as sputtering and pulsed-laser-deposition (PLD). In our oxide-film studies, we have employed PLD (PVD Products, Inc.) with a $\mathrm{KrF}$ excimer laser (Lambda Physik $\mathrm{GmbH}$ ) at a wavelength of $248 \mathrm{~nm}$ for source material ablation. The chosen ablation targets were $\mathrm{Ga}_{2} \mathrm{O}_{3}$ and $\mathrm{ZnO}$ for $\mathrm{GZO}, \mathrm{Al}_{2} \mathrm{O}_{3}$ and $\mathrm{ZnO}$ for $\mathrm{AZO}$, and $\mathrm{In}_{2} \mathrm{O}_{3}$ and $\mathrm{SnO}_{2}$ for ITO. The targets were purchased from the Kurt J. Lesker Corp. with purities of $99.99 \%$ or higher. The required composition of the deposited film was achieved by alternating the laser ablation over two different targets with an appropriate number of pulses on each target. A single cycle consisting of a few laser pulses on each target was repeated many times until the desired film thickness was achieved. The number of pulses in each cycle was designed to be small enough so that the effective layer thickness deposited in a single cycle would be less than a few atomic layers. This ensured a homogeneous mixture of the constituent materials in the final film. All the films were grown in an oxygen ambient with an oxygen partial pressure of $0.4 \mathrm{mTorr}(0.053 \mathrm{~Pa})$ or lower. The substrate was heated to temperatures around $50-100{ }^{\circ} \mathrm{C}$ during deposition. The deposition conditions were optimized to achieve highest possible carrier concentration and lowest possible losses. High carrier concentration produces metal-like properties for larger frequency-range in the near-IR and low loss would enable high performance devices. The optimization curves for GZO, ITO and AZO are shown in Fig. 1. The optical characterization of the thin films was performed using a spectroscopic ellipsometer (V-VASE, J. A. Woollam). The dielectric function was retrieved by fitting

\#151151 - \$15.00 USD Received 15 Jul 2011; revised 29 Aug 2011; accepted 30 Aug 2011; published 6 Sep 2011

(C) 2011 OSA

1 October 2011 / Vol. 1, No. 6 / OPTICAL MATERIALS EXPRESS 1093 

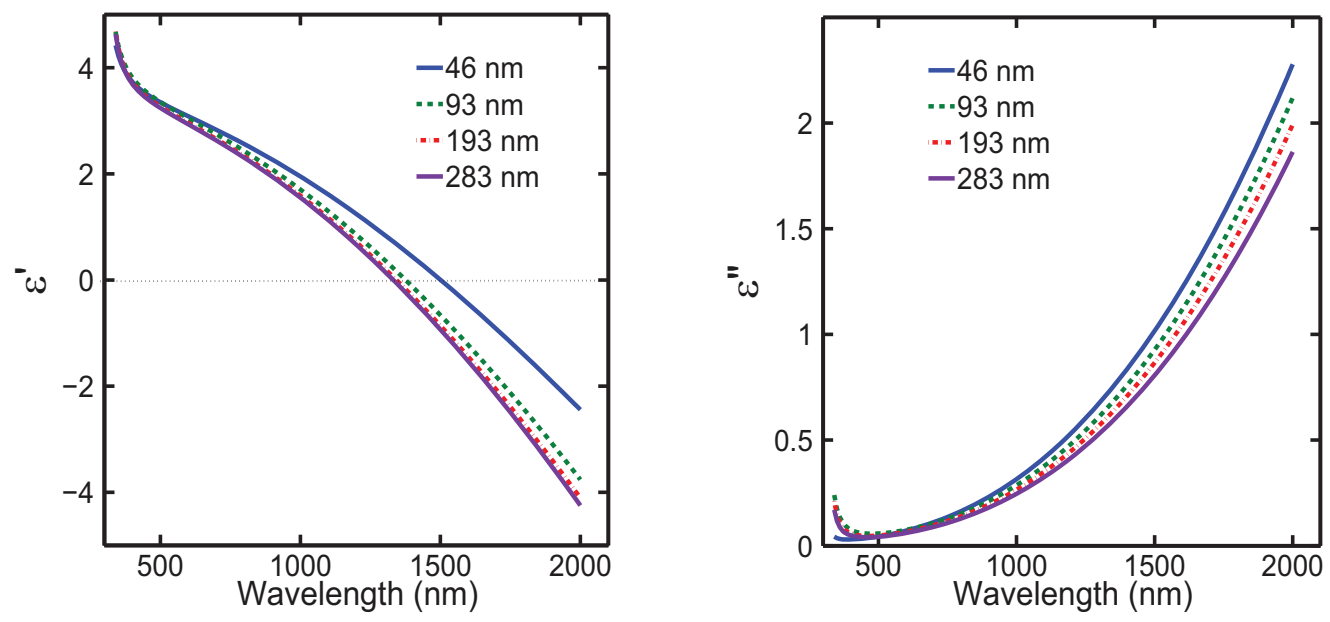

Fig. 2. Optical properties of GZO thin films with different thicknesses deposited on glass substrates. The films were deposited under identical conditions except for the duration of deposition.

a Drude+Lorentz oscillator model to the ellipsometry data.

The cross-over frequency $\left(\omega_{c}\right)$ is defined as the frequency at which the real permittivity of the material crosses zero. Since $\omega_{c}$ is directly proportional to plasma frequency $\left(\omega_{p}\right)$ and $\omega_{p}$ is proportional to the square of carrier concentration, Fig. 1 depicts the carrier concentration trend as a function of film composition. The carrier concentration reaches a maximum near the solid solubility limit of the dopant and drops slightly for higher dopant concentrations [13]. Damping losses show small dependence on dopant concentration for ITO and GZO films. AZO films show lower losses for higher dopant concentration, most likely due to improved crystallinity of the film [14]. While the highest cross-over frequency achieved for AZO is about $0.7 \mathrm{eV}$, the same for GZO and ITO is around $0.9 \mathrm{eV}$. The differences in the properties of AZO, GZO and ITO occur as a result of the differences in their dopant solid-solubility limits and doping efficiencies $[13,15]$. The substrate temperature and oxygen partial pressure during deposition of these films played significant roles in achieving the highest possible carrier concentration. Oxygen-vacancy defects in $\mathrm{ZnO}$ are known to provide additional carriers [16]. In the fabricated films, it was found that higher oxygen partial pressure reduced carrier concentration due to reduced number of oxygen-vacancy defects. Another parameter that influences carrier concentration is substrate temperature. It has been shown that low substrate temperature increases losses and high temperature reduces carrier concentration [17]. Thus, the optimum values for substrate temperature and oxygen partial pressures were found through multiple deposition experiments (Fig. 1). In an attempt to further increase the carrier concentration by post-processing steps, we found that thermal annealing can only lead to decrease in carrier concentration. Therefore, no post-processing steps were carried out on the films.

Owing to their non-stoichiometric nature, TCO films are known to exhibit thicknessdependent properties $[18,19]$. This is because the interface with the substrate can have many carrier trap states, which reduce the net carrier concentration. Thus, the net carrier concentration in the film depends on the thickness (volume to surface ratio) of the film [20,21]. The losses also depend on the thickness because scattering is dependent on the microstructure of the film

\#151151 - \$15.00 USD Received 15 Jul 2011; revised 29 Aug 2011; accepted 30 Aug 2011; published 6 Sep 2011

(C) 2011 OSA

1 October 2011 / Vol. 1, No. 6 / OPTICAL MATERIALS EXPRESS 1094 

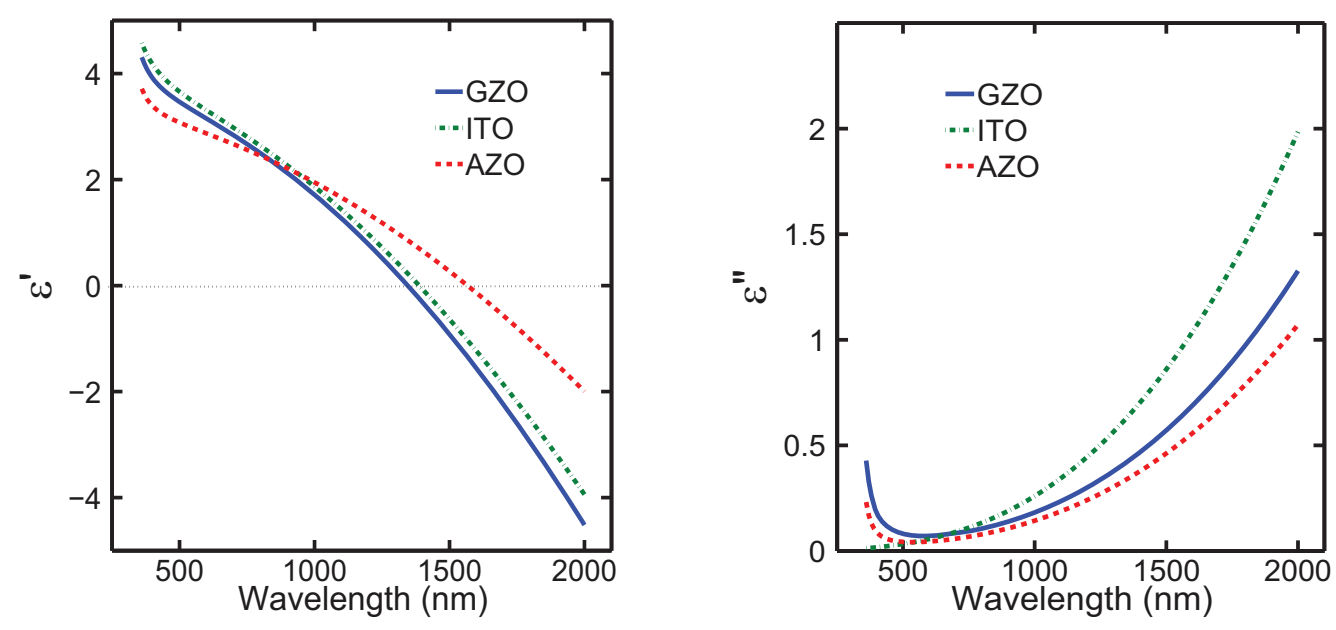

Fig. 3. Comparison of the optical properties of pulsed laser deposited TCO films with the smallest cross-over wavelengths. The films were deposited onto glass substrates at $100{ }^{\circ} \mathrm{C}$ (AZO and ITO) and $50{ }^{\circ} \mathrm{C}(\mathrm{GZO})$ with oxygen partial pressures of $0.4 \mathrm{mTorr}$.

and the microstructure is thickness dependent $[18,19]$. In many nanoplasmonic devices, thin film structures are used as building blocks, and therefore it is necessary to understand how the optical properties of TCO thin films depend on their thickness. We have studied the thicknessdependent optical properties of GZO thin films on glass substrates. Figure 2 shows the dielectric function of GZO films with different thicknesses. The films with thicknesses greater than about $50 \mathrm{~nm}$ exhibit very little thickness dependence in their optical properties.

As a comparison of the three different TCOs considered in our study, in Fig. 3 we plot the optical properties of AZO, GZO and ITO films. The plots correspond to the TCO films with the lowest cross-over wavelengths and lowest losses. Notably, AZO offers the lowest Drude damping, but it also has the lowest $\omega_{c}$ (and hence the longest cross-over wavelength). GZO and ITO can produce cross-over wavelength as low as $1.2 \mu \mathrm{m}$. However, Drude damping in GZO is slightly higher than that in AZO and lower than that in ITO.

\subsection{Transition metal nitrides}

Many of the transition-metal nitrides are known to exhibit metallic properties in the visible frequencies [22]. Their optical properties largely depend on the deposition conditions because of their non-stoichiometric nature. As a general trend, metal-rich films are metallic and nitrogenrich films are dielectric. As an exception, TiN is metallic both when metal-rich and when nitrogen-rich. However, the carrier concentration declines from metal-rich to nitrogen-rich films. The nitride films are also sensitive to the substrate because they show textured growth on some specific substrates. For example, c-sapphire and $\mathrm{MgO}$ substrates can be low latticemismatched substrates [23,24], promoting layer-by-layer crystalline growth. On the other hand, glass as a substrate produces polycrystalline or amorphous films, which exhibit quite different properties than single-crystal films. Importantly, the losses are significantly smaller for crystalline films than those of amorphous or polycrystalline films [25].

In our study of nitrides for plasmonic applications, we have employed DC reactive sputtering to deposit thin films of metal nitrides. Pure metal targets $(99.995 \%)$ were sputtered in

\#151151 - \$15.00 USD Received 15 Jul 2011; revised 29 Aug 2011; accepted 30 Aug 2011; published 6 Sep 2011

(C) 2011 OSA

1 October 2011 / Vol. 1, No. 6 / OPTICAL MATERIALS EXPRESS 1095 

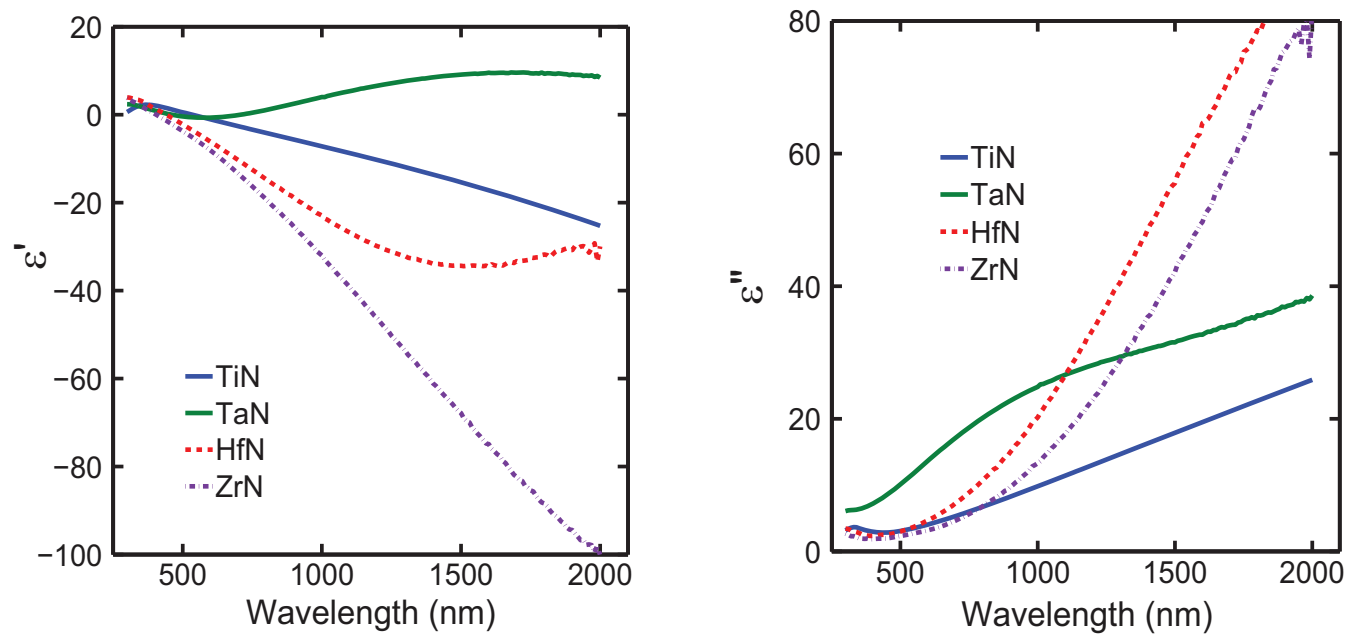

Fig. 4. Dielectric function retrieved from spectroscopic ellipsometry measurements on thin films of TiN, TaN, HfN and $\mathrm{ZrN}$ deposited on c-sapphire. The substrate temperature during deposition was $800{ }^{\circ} \mathrm{C}$, and the chamber pressure was 5 mTorr. The flow ratios of $\mathrm{N}_{2}: \mathrm{Ar}$ were $6 \mathrm{sccm}$ : $4 \mathrm{sccm}$ for TaN and TiN films and $2 \mathrm{sccm}: 8 \mathrm{sccm}$ for the rest. The sputter power was $150 \mathrm{~W}$ for Ta and $\mathrm{Hf}$ targets and $200 \mathrm{~W}$ for the rest.

a nitrogen-argon ambient with partial pressures of nitrogen and argon adjusted by controlling their flow rates. The base pressure was about $4 \times 10^{-7}$ Torr or lower, and the sputtering pressure was held at 5 mTorr. The substrate was either glass or c-axis oriented sapphire and was heated to an elevated temperature during deposition. Films about 30-50 nm thick were deposited on the substrates, and their optical characterization was performed using a spectroscopic ellipsometer. The optical constants were retrieved using a Drude+Lorentz oscillator model accounting for intra-band and inter-band electronic contributions. The dielectric functions of thin films of TiN, $\mathrm{TaN}, \mathrm{ZrN}$ and $\mathrm{HfN}$ deposited on sapphire substrates at $800^{\circ} \mathrm{C}$ are shown in Fig. 4. The nitrogen to argon flow ratio was maintained during deposition at $2 \mathrm{sccm}: 8 \mathrm{sccm}$ for $\mathrm{HfN}, \mathrm{ZrN}$ films and $6 \mathrm{sccm}: 4 \mathrm{sccm}$ for TiN and TaN films. These conditions were found to produce nitride films that are metallic in the visible frequencies. Figure 4 shows that TiN is metallic for wavelengths longer than about $500 \mathrm{~nm}$. $\mathrm{HfN}$ and $\mathrm{ZrN}$ have cross-over wavelengths around $430 \mathrm{~nm}$. TaN is metallic in the visible range only and becomes dielectric for longer wavelengths. This behavior is probably due to the nitrogen-rich deposition conditions. We note that the losses in these films are not small, partly because of the presence of inter-band transition losses. However, in the regions where inter-band losses are absent, the losses are mainly due to Drude damping. From Fig. 4, TiN and $\mathrm{ZrN}$ appear to be low-loss candidates for alternative plasmonic materials.

As stated previously, the optical properties of nitrides depend significantly on whether they are nitrogen-rich or metal-rich. In order to study this, $\mathrm{ZrN}$ thin films were deposited on glass substrates with two different nitrogen partial pressures during deposition. Figure 5 shows the optical constants of these $\mathrm{ZrN}$ films. The optical properties turn from metallic for a nitrogenpoor ambient to dielectric for a nitrogen-rich ambient. This behavior is typical to many ceramic nitrides owing to their non-stoichiometric compositions [22]. This drastic change in film properties can be useful in building plasmonic devices where the properties of the material components need to be tuned or graded.

\#151151 - \$15.00 USD Received 15 Jul 2011; revised 29 Aug 2011; accepted 30 Aug 2011; published 6 Sep 2011

(C) 2011 OSA

1 October 2011 / Vol. 1, No. 6 / OPTICAL MATERIALS EXPRESS 1096 


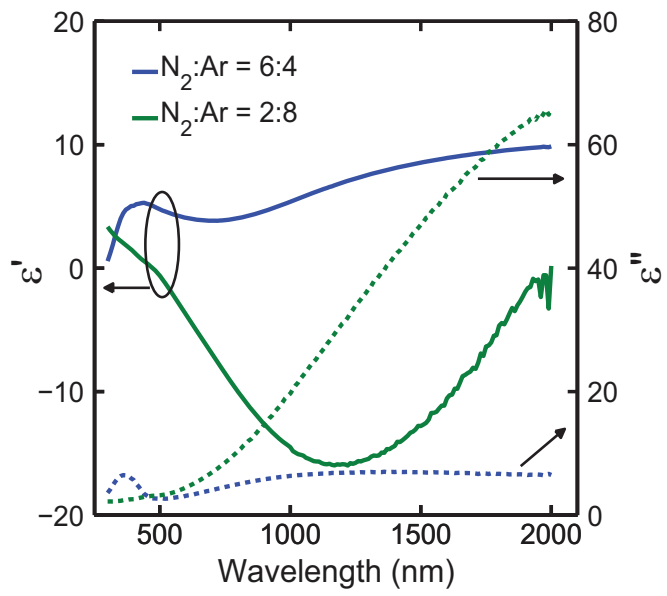

Fig. 5. Optical constants for $\mathrm{ZrN}$ films deposited on glass at different $\mathrm{N}_{2}$ :Ar flow ratios $(\mathrm{sccm} / \mathrm{sccm})$. The sputtering pressure was held at $5 \mathrm{mTorr}$ and the substrate temperature during deposition was $350{ }^{\circ} \mathrm{C}$. While the $\mathrm{ZrN}$ film deposited in a nitrogen-rich ambient shows dielectric properties, the film deposited in a nitrogen-poor ambient shows metallic properties.

In this section the details of fabrication and their effects on the optical properties of oxides and nitrides were presented. The findings of this section shed light on the applicability of these new materials for plasmonic and metamaterial applications. The findings also help us in assessing the various advantages and disadvantages from the viewpoint of plasmonic devices as well as in terms of nanofabrication and integration. The following section discusses some of these important implications of the new class of plasmonic materials.

\section{Discussions}

Novel devices made with metamaterials and transformation optics such as superlenses [26], hyperlenses [27] and cloaks [28] require special conditions to perform efficiently. One of these conditions is that the real part of permittivity of the metallic and dielectric components should be nearly the same magnitude [29]. In the visible and near-IR frequencies, this condition is not sufficiently met by conventional metals. However, alternative plasmonic materials described in the previous section do meet this condition. In Fig. 6a we plot the real part of permittivity for nitrides and oxides together with those of silver and gold [30]. The arrows show the range in which nitrides and oxides are metallic. While TCOs are useful as plasmonic materials in the near-IR, nitrides are useful in the visible range. In their respective metallic ranges, both oxides and nitrides have much smaller magnitudes of real permittivity compared to those of either gold or silver.

Another important disadvantage of conventional metals is high loss. Conventional metals have very high carrier concentrations, which in turn makes their plasma frequencies very large. A large plasma frequency produces a large imaginary permittivity, which translates to large loss. Alternative plasmonic materials have lower carrier concentrations and, hence, smaller losses (see Fig. 6b). In particular, TCOs exhibit losses nearly five times smaller than that of the best metal $(\mathrm{Ag})$ in the near-IR. This makes TCOs a good choice for many metamaterial and plasmonic applications in the near-IR and mid-IR ranges [31,32]. However, the losses in

\#151151 - \$15.00 USD Received 15 Jul 2011; revised 29 Aug 2011; accepted 30 Aug 2011; published 6 Sep 2011

(C) 2011 OSA

1 October 2011 / Vol. 1, No. 6 / OPTICAL MATERIALS EXPRESS 1097 

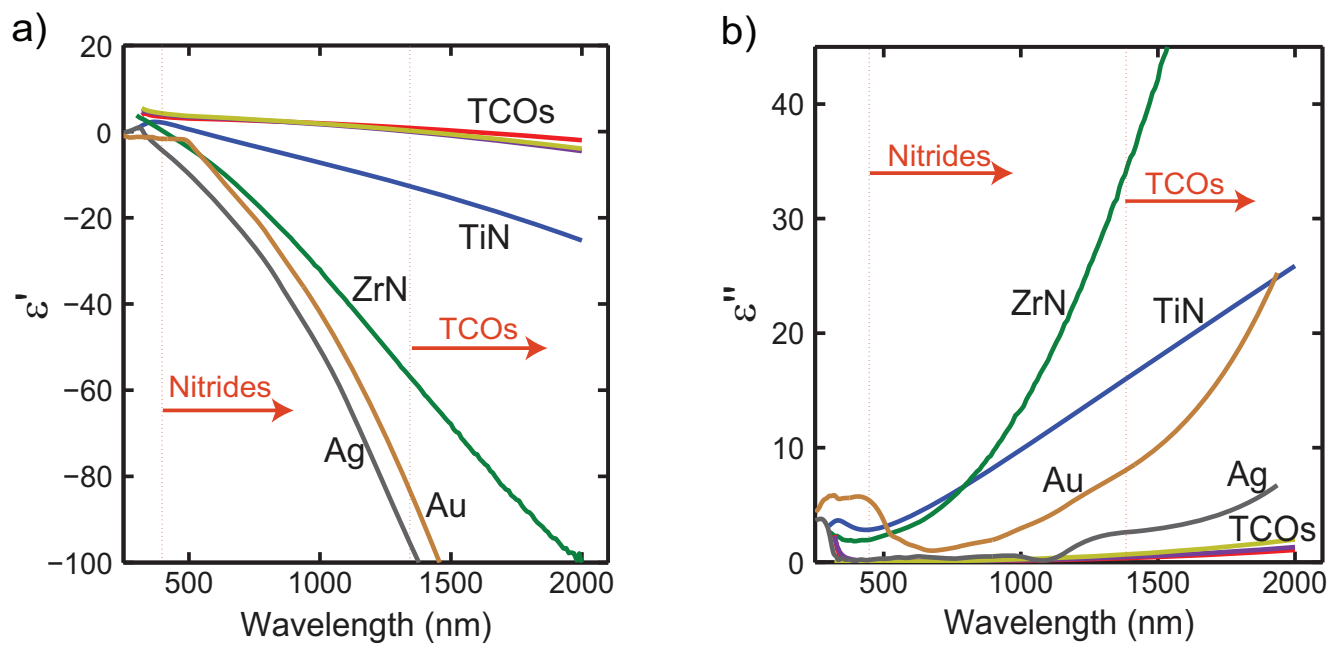

Fig. 6. Comparison of optical properties of alternative plasmonic materials with those of conventional metals. Optical constants of low loss thin films of TiN, $\mathrm{ZrN}$ and TCOs (AZO, GZO and ITO) are plotted along with those of gold and silver taken from [30]. The arrows show the wavelength ranges in which nitrides and TCOs are respectively metallic. Panel a) shows that TCOs and nitrides have smaller negative permittivity values than those of metals, while b) compares losses and shows that losses in TCOs are many times smaller than those in either gold or silver. The losses in nitrides are slightly higher than metals due to inter-band transitions at the cross-over.

transition-metal nitrides are not as low as TCOs because of larger Drude damping and interband losses. Comparing Drude-damping in metals and nitrides, metals have lower losses. However, upon fabrication of metallic nanostructures, the Drude-damping in metals increase many times due to imperfections such as roughness and grain-boundary scattering [33]. Such problems can be minimized in nitrides because they can be grown as crystalline layers on substrates like sapphire and hence, can posses very small surface roughness [34]. Thus, in the frequency range where inter-band losses are absent, nitrides can be good alternatives to metals.

Another requirement of novel devices is tunability in the constituent material properties. Tunability can be static or dynamic depending on the application. A gradient in the properties of the constituent materials would be essential in designing many novel devices [29,35]. Conventional metals such as gold and silver cannot be tuned. Hence, they seriously limit the efficient implementation of new plasmonic and metamaterial devices. Alternative plasmonic materials do not pose such problems. Their properties are strongly dependent on the processing conditions; thus, they provide a useful method of tuning the device properties.

An additional advantage of alternative plasmonic materials lies in their ease of fabrication and integration. Fabrication techniques such as chemical vapor deposition, atomic layer deposition and molecular beam epitaxy can be employed to produce oxide and nitride films of high quality. Furthermore, employing lattice-matched systems allows hetero-epitaxy, which could produce high-performance, monolithic devices. For example, AZO and GZO on sapphire or $\mathrm{ZnO}$ substrates could result in superlattice/monolithic devices [36-38]. Similarly, nitrides have good lattice match with sapphire or $\mathrm{MgO}$ substrates [23, 24]. It is important to note that conventional metals such as gold and silver are not compatible with standard CMOS processes. However, this limitation can be completely overcome by alternative plasmonic materials such

\#151151 - \$15.00 USD Received 15 Jul 2011; revised 29 Aug 2011; accepted 30 Aug 2011; published 6 Sep 2011

(C) 2011 OSA

1 October 2011 / Vol. 1, No. 6 / OPTICAL MATERIALS EXPRESS 1098 
as TaN and TiN, which are CMOS-compatible and have been shown to be useful as gate metals in CMOS transistors [6].

As a summary, alternative plasmonic materials offer many advantages over conventional metals for plasmonic and metamaterial devices. Each material system considered here has its own set of advantages for a specific class of devices operating in a particular part of the optical spectrum. An appropriate choice from the subset of materials presented here could enable highperformance devices in the optical frequency range.

\section{Conclusion}

Alternative plasmonic materials have many advantages when replacing conventional metals for plasmonic and metamaterial applications. However, the properties of these alternative materials are sensitive to deposition conditions and require optimization. Optimized films of transparent conducting oxides (TCOs) such as AZO, GZO and ITO are low-loss alternatives to metals in the near-IR, where they could enable high-performance devices such as hyperlenses and superlenses. In the visible range, nitrides can be alternatives to metals although nitrides have losses slightly higher than conventional metals. Nitrides also offer many fabrication and integration advantages over conventional materials such as gold and silver.

\section{Acknowledgments}

We thank Prof. Vladimir M. Shalaev and Prof. Timothy D. Sands for their support. We thank Prof. Thomas A. Klar, Prof. Johannes Pedarnig, Prof. Peter Schaaf and Dr. Marius-Aurel Bodea for helpful discussions. This work was supported in part from ONR-MURI grant N00014-101-0942.

\#151151 - \$15.00 USD Received 15 Jul 2011; revised 29 Aug 2011; accepted 30 Aug 2011; published 6 Sep 2011

(C) 2011 OSA

1 October 2011 / Vol. 1, No. 6 / OPTICAL MATERIALS EXPRESS 1099 